\title{
Influence of permeability anisotropy on heat transfer and permeability evolution in geothermal reservoir
}

\author{
Janim Joshua Ijeje ${ }^{1}$, Quan Gan $^{1 \oplus *}$, Jianchao Cai ${ }^{2}$ \\ ${ }^{1}$ School of Geosciences, University of Aberdeen, Aberdeen, UK \\ ${ }^{2}$ Hubei Subsurface Multi-scale Imaging Key Laboratory, Institute of Geophysics and Geomatics,
}

China University of Geosciences, Wuhan 430074, P. R. China

(Received August 25, 2018; revised September 4, 2018; accepted September 5, 2018; available online September 12, 2018)

\section{Citation:}

Ijeje, J.J., Gan, Q., Cai, J. Influence of permeability anisotropy on heat transfer and permeability evolution in geothermal reservoir. Advances in Geo-Energy

Research, 2019, 3(1): 43-51, doi:

10.26804/ager.2019.01.03.

Corresponding author:

*E-mail: gan.quan@abdn.ac.uk

Keywords:

Permeability anisotropy

fracture aperture

thermal drawdown

geothermal reservoir

\begin{abstract}
:
Extracting heat energy from geothermal reservoirs essentially relies on circulating cold fluid within fractured hot rocks. The intrinsic anisotropic permeability in the reservoir directly affects the path of flow and the associated thermal drawdown from cooling procedure. Consequently, each individual component including thermal, hydraulic, and mechanical field needs to be considered, to evaluate the influence of permeability anisotropy on the thermal production and evolution of rock properties. In this work, the fully implicit coupled simulator TFReact was successfully implemented to generate results prototypical of an enhanced geothermal system. Anisotropic permeability values were generated from the variation of fracture spacing at three orthogonal principal directions, with identical initial fracture aperture. Five case scenarios of permeability anisotropy were simulated to evaluate the influence of anisotropic thermal drawdown in triggering permeability evolution. Analysis of the propagation of the thermal front from injector to producer indicated that low anisotropic permeability values will lead to late cold water breakthrough at producer, slower migration rate and wider sweep area than high anisotropic permeability values. Isotropic permeability scenario showed a lower thermal gradient profile, comparing against the scenarios of anisotropic permeability. The anisotropic value of 0.01 produced the highest power output, while isotropic permeability generated the least power output. Induced thermal stress resulted an unloading response by reducing compressive normal stress in sub-horizontal direction, and thereby increase fracture aperture in sub-horizontal direction. Invariably, the induced thermal expansion stress increased the compressive stress and reduced fracture aperture and permeability. After the same timing of injectionproduction cycle, the highest anisotropic permeability scenario resulted a factor of 2.5 increment in evolving fracture permeability, while lowest anisotropic permeability scenario lead to a factor of 0.35 decrease in changing fracture permeability. The generated thermal output suggested the most favorable strategy in maximizing thermal sweep across the reservoir, by prompting thermal transfer normal to direction of injector-producer.
\end{abstract}

\section{Introduction}

In deep enhanced geothermal systems (EGS), fractures are ubiquitously distributed within reservoirs, serving as major conduits for fluid circulation. The rock matrix provides most of the heat energy storage within the reservoir (Pruess, 1983). Fluid and heat are transferred by convection into the high permeable fracture network (Watanabe and Takahashi, 1995; Jahediesfanjani and Civan, 2006). The transfer modes of fluid flow and heat transfer between rock matrix and fractures play a vital role on the performance of the enhanced geothermal system and other geological applications such as: radioactive waste repositories, $\mathrm{CO}_{2}$ sequestration reservoirs, petroleum reservoirs, and fracturing and faulting of rocks (Rutqvist and Stephansson, 2003).

The evolution of heat transfer is significantly affected by the pattern of fracture networks in enhanced geothermal system (Gan, 2016a). Even given the same equivalent magnitude of permeability, but the difference in density/spacing of fractures will result entire different heat transfer mode. The permeability of most geo-engineering reservoir, including geothermal reservoirs, in all three principal directions is commonly anisotropic (Snow, 1969). The anisotropic permeability of a reservoir can either improve or reduce heat transfer across the reservoir by conduction or advection (Gan, 2016b). Also, 


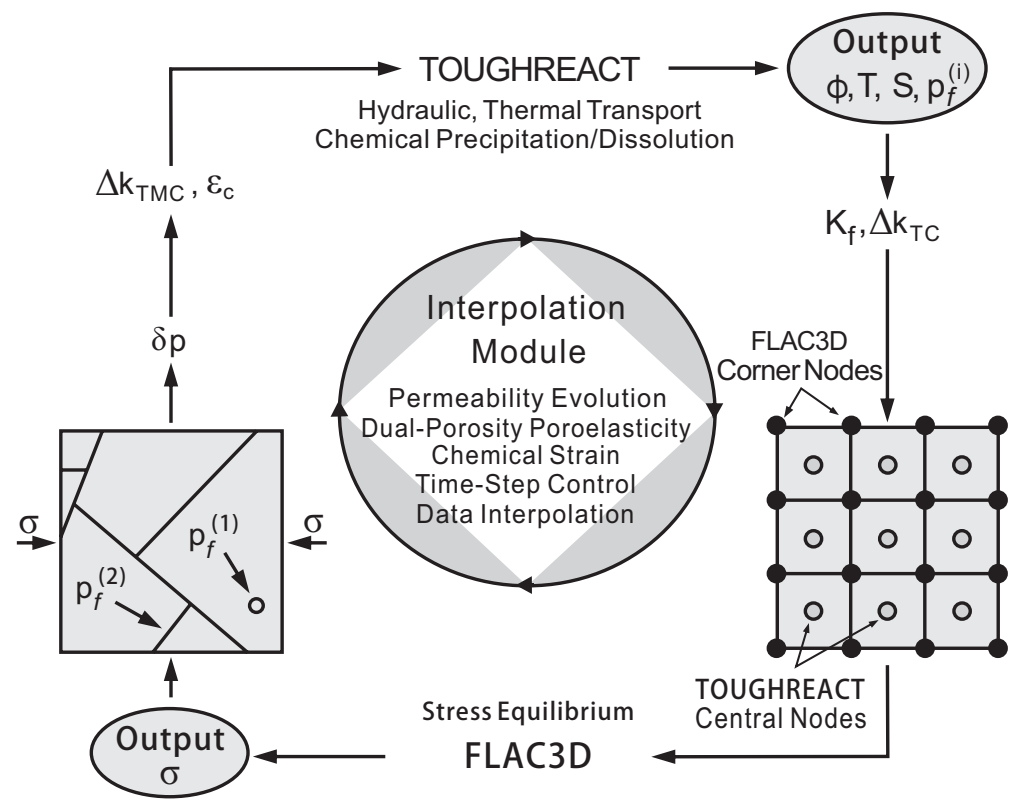

Fig. 1. Flowchart and coupling interaction between TOUGHREACT, FLAC3D and interpolation module (Taron et al., 2009).

anisotropic permeability significantly affects the hydraulic and mechanical properties of fractured rocks in response to thermal, hydraulic, mechanical, and chemical forces (Taron et al., 2009). Fluid flow within the fracture network determines the lifespan of a geoengineering project. The fracture permeability and porosity control the rate of fluid flow within the fracture network. Changes in effective stress and thermal expansion induce mechanical stresses which result in gaping or sealing of fractures which in turn changes the fracture permeability (Min et al., 2008). A better understanding of the influence of anisotropic permeability on heat transfer between reservoir rocks and fractures and fluid flow forms an integral part to the success of a geoengineering project.

Several analytical and semi-analytical models have been developed to predict the heat transfer in fractured rocks (Bodvarsson, 1969; Gringarten et al., 1975; Bodvarsson and Tsang, 1982; Elsworth, 1989; Kolditz and Clauser, 1998). Taron and Elsworth (2010) investigated the effect of spatial heterogeneity on temperature and permeability evolution between an injection and withdrawal wells using numerical models. Subsequently, Gan and Elsworth (2014a) analysed the effects of injection temperature, changes in fracture permeability, and fracture spacing on timing and magnitude of seismic events. The results revealed that fracture spacing controls the rate of heat energy transfer and thermal drawdown within a geothermal reservoir. The smaller the fracture spacing will lead to a more comprehensive thermal drawdown and same for thermal energy recovery. This study aims to investigate the effect of anisotropic permeability variation on heat transfer and identify the optimal anisotropic permeability value that would produce maximum heat transfer between the rock matrix and fluid-filled fracture network. Also, the study aims to investigate the evolution of anisotropic permeability and the response of anisotropic permeability to the induce thermal stress.

\subsection{Simulation mechanism}

The TOUGHREACT-FLAC3D (TFReact v2.0) simulator was used for the numerical simulation. The TOUGHREACTFLAC3D simulator couples the thermal $(\mathrm{T})$, hydraulic $(\mathrm{H})$ and chemical (C) precipitation/dissolution capabilities of TOUGHREACT with the mechanical (M) framework of FLAC3D to examine THMC processes in deformable, dual medium fractured porous media (Taron et al., 2009). FLAC3D solved the geomechanical stress-strain equations (Itasca, 2009), whereas the TOUGHREACT solved the multiphase flow and heat transport equations. Both codes use the same mesh, and the TOUGHREACT central node data is interpolated to corner node information as input for FLAC3D (Fig. 1). An external coupling module referred to as the "interpolation module" links the TOUGHREACT and FLAC3D together, by the constitute models of evolution of permeability, porosity, poroelasticity, and element data interpolation, etc. This module is a Fortran 90 executable capable of rendering data outputs from one simulator as input for the other. The module also calculates the pressure and temperature dependent thermodynamic compressibility of reservoir fluids, dual porosity poroelasticity response to stress, applies aperture changes to bulk permeability field and evolves fracture aperture due to mechanical and chemical processes (Taron and Elsworth, 2009). The accuracy of coupling linking FLAC3D and Tough in reflecting the evolution fluid transport and mechanical properties has been extensively benchmarked in previous works (Taron, 2009, 2010; Gan, 2014a, 2014b, 2016).

\subsection{Governing equations}

Within the framkework of Tough-FLAC3D, the fluid flow, stress and deformation, and thermal transfer are handled separately in Tough and FLAC3D. The mass conservation 
equation governing the flow in permeable medium are written in the following form (Pruess, 2004):

$$
\frac{d}{d t} \int_{V_{n}} M d V_{n}=\int_{\Gamma_{n}} F \cdot n d \Gamma_{n}+\int_{V_{n}} q d V_{n}
$$

The above integration described the flow system over an abitrary domain $V_{n}$, which is bounded by the closed surface $\Gamma_{n}$. The equation indicates the balance between the rate of mass change in particular volume $V_{n}$ is equal to net inflow across the surface of volume $V_{n}$, and plus the mass inflow from the sink and sources. The symbol $M$ in the accumulation term represents the mass per volume for particular fluid. $F$ denotes the mass flux, and $q$ represents the sinks and sources. $n$ is a normal vector on surface element $d \Gamma_{n}$ pointing into $V_{n}$.

The differential equations governing heat transfer in the fracture are based on the balance of heat energy in the control volume of fractures, defined as:

$$
\rho_{w} c_{w} \frac{\partial T_{w}(x, t)}{\partial t}=-v \rho_{w} c_{w} \frac{\partial T_{w}(x, t)}{\partial x}+\left.\frac{2 K_{r}}{b} \frac{\partial T_{r}(x, z, t)}{\partial z}\right|_{z=\frac{b}{2}}
$$

where $v$ is the fluid velocity $(\mathrm{m} / \mathrm{s}), T_{w}(x, t)$ is the temperature of water, $T_{r}(x, z, t)$ is the temperature of the rock matrix, $b$ is the fracture aperture $(\mathrm{m}), c_{w}$ is the heat capacity of water $\left(\mathrm{J} / \mathrm{kg} /{ }^{\circ} \mathrm{C}\right), \rho_{w}$ is the density of water, and $K_{r}$ is the thermal conductivity of the rock $\left(\mathrm{J} / \mathrm{s} / \mathrm{m} /{ }^{\circ} \mathrm{C}\right)$.

The temperature of the rock matrix is governed by the heat conduction equation:

$$
\frac{\partial^{2} T_{r}(x, z, t)}{\partial z^{2}}=\frac{\rho_{r} c_{r}}{K_{r}} \frac{\partial T_{r}(x, z, t)}{\partial t}
$$

where $\rho_{r}$ is the density of the rock matrix, and $c_{r}$ is the heat capacity of the rock.

\subsection{Constitutive model of permeability}

The anisotropic permeability in this work is represented by three orthogonal fracture planes ( $x, y$ and $z$-axes), each has its unique fracture compression and dilation different from the others. Anisotropic permeability is a function of the current pressure and temperature conditions and the normal stress acting on each plane (Taron and Elsworth, 2010). Fracture permeability is defined by an orthogonal set of fractures based on the cubic law (Witherspoon et al., 1980):

$$
k_{f}=\frac{b^{3}}{12 s}
$$

where $b$ is the fracture aperture, $s$ is the fracture spacing, and $k_{f}$ is corresponded fracture permeability. Fracture aperture under an applied effective normal stress, $\sigma$, may be defined empirically by a non-linear relationship that accommodates the observed stiffening of fractures with closure (Min et al., 2008; Taron and Elsworth, 2009; Taron et al., 2009):

$$
b_{m}=b_{m r}+\left(b_{m o}-b_{m r}\right) e^{-\omega\left(\sigma-\sigma_{0}\right)}
$$

where $b_{m}$ is the fracture aperture resulting from mechanical effects, $b_{m o}$ is the aperture under no mechanical stress, $b_{m r}$ is the residual aperture at maximum mechanical loading, $\sigma_{0}$ is the effective stress at which zero deformation occurs (usually zero), and $\omega$ is a constant that defines the non-linear stiffness of the fracture. This constitutive model of dynamic aperture defined the changes in effective stress $\sigma$ can modify fracture apertures which in turn changes permeability. Since the reservoir geometry in this work is a pseudo-3D doublet, and the thickness in the $y$ direction is negiligble compared to the dimensions of $x$ and $z$-axes, the parameter of anisotropic permeability $\eta$ is defined as:

$$
\eta=\frac{k_{f x}}{k_{f z}}
$$

where $k_{f x}$ and $k_{f z}$ are the fracture permeability in the $x$ and $z$ direction respectively.

\subsection{Model description}

Fig. 2 represents the reservoir configuration with gridding used in this study. The behaviour of heat transfer processes within the fractured porous medium was examined with conditions similar to an enhanced geothermal system. The dimension of the reservoir $(1320 \mathrm{~m} \times 15 \mathrm{~m} \times 420 \mathrm{~m})$ created a pseudo-3D doublet which contained three sets of orthogonal fractures. The fractures served as the effective pathway for fluid circulation within the reservoir. Two radially graded mesh was generated $480 \mathrm{~m}$ apart to represent the injection and withdrawal wells. The initial rock and fluid temperature are homogenous at $200{ }^{\circ} \mathrm{C}$ across the reservoir with a homogeneous reservoir pressure of $13.8 \mathrm{MPa}$. The initial boundary stresses along the remote $x, y$ and $z$-axes are assigned at $35 \mathrm{MPa}, 45 \mathrm{MPa}$, and $35 \mathrm{MPa}$ respectively. The initial stresses were allowed to equilibrate with the initial pore pressure (13.8 MPa), and the model boundaries were set to no flow boundaries with applied constant stresses. Cold water was injected at a constant pressure of $21.8 \mathrm{MPa}$ with $70^{\circ} \mathrm{C}$, and the hot water was collected through the withdrawal well, operated at a bottom-hole pressure of $10.8 \mathrm{MPa}$. Table 1 shows the adopted material properties of the fractured rock medium in

Table 1. Rock properties used in simulation.

\begin{tabular}{lll}
\hline Parameters & Units & Values \\
\hline Poission's ratio & - & 0.22 \\
Friction angle & $\circ$ & 30 \\
Cohesion & $\mathrm{MPa}$ & 0.05 \\
Tensil strength & $\mathrm{MPa}$ & $10^{4}$ \\
Dilation angle & $\circ$ & 10 \\
Thermal expansion & ${ }^{\circ} \mathrm{C}^{-1}$ & 12 \\
Solid density & $\mathrm{kg} / \mathrm{m}^{3}$ & 2700 \\
Bulk modulus & $\mathrm{GPa}$ & 8 \\
Biot Coeff. & - & 0.8 \\
Fracture/matrix porosity & - & $0.5 / 0.02$ \\
Matrix permeability & $\mathrm{m}^{2}$ & $10^{-18}$ \\
\hline
\end{tabular}




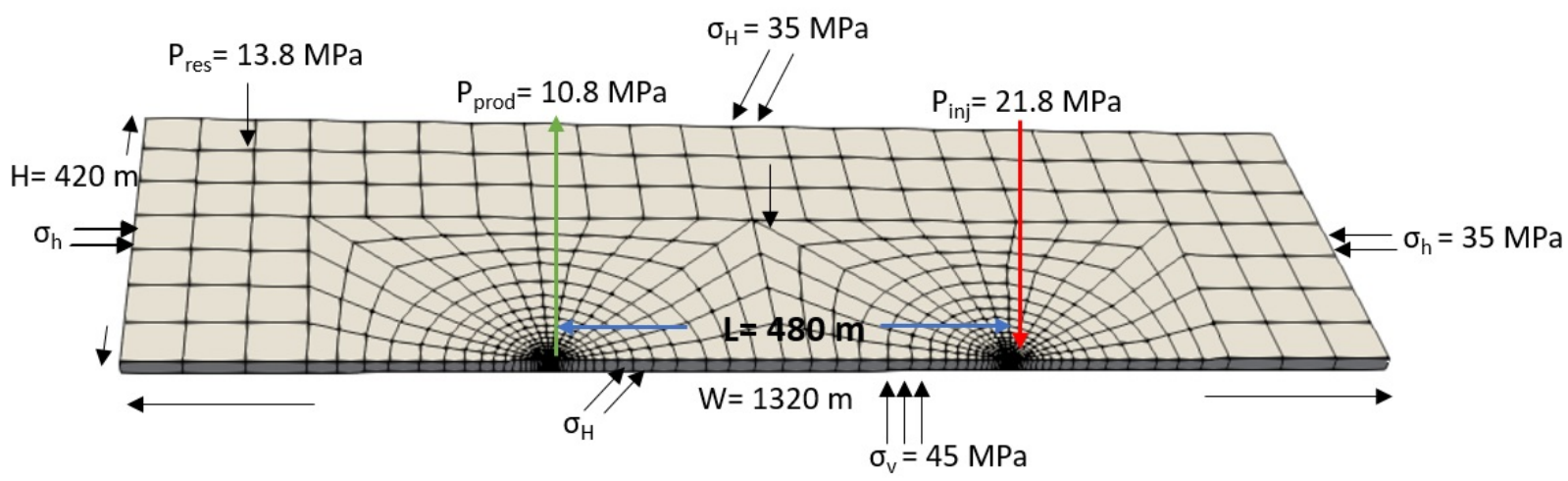

Fig. 2. Model geometry of geothermal reservoir with the applied stress boundary conditions and the initial pressure conditions, injection well (right) and production well are placed in the lower boundary with $480 \mathrm{~m}$ separation.

Table 2. TFReact initial permeability and corresponding fracture parameterisation for each case scenarios.

\begin{tabular}{llllllllll}
\hline Cases & $S_{x}$ & $S_{y}$ & $S_{z}$ & $b_{m r}(\mathrm{~m})$ & $b_{m o}(\mathrm{~m})$ & $\begin{array}{l}\omega \\
(1 / \mathrm{MPa})\end{array}$ & $K_{x}\left(\mathrm{~m}^{2}\right)$ & $K_{z}\left(\mathrm{~m}^{2}\right)$ & $\eta$ \\
\hline 1 & 0.1 & 10 & 10 & $4.004 \mathrm{e}-4$ & $1.602 \mathrm{e}-3$ & 0.218 & $5.84 \mathrm{e}-11$ & $5.84 \mathrm{e}-13$ & 100 \\
2 & 1 & 10 & 10 & $4.004 \mathrm{e}-4$ & $1.602 \mathrm{e}-3$ & 0.218 & $5.84 \mathrm{e}-12$ & $5.84 \mathrm{e}-13$ & 10 \\
3 & 10 & 10 & 10 & $4.004 \mathrm{e}-4$ & $1.602 \mathrm{e}-3$ & 0.218 & $5.84 \mathrm{e}-13$ & $5.84 \mathrm{e}-13$ & 1 \\
4 & 10 & 10 & 1 & $4.004 \mathrm{e}-4$ & $1.602 \mathrm{e}-3$ & 0.218 & $5.84 \mathrm{e}-13$ & $5.84 \mathrm{e}-12$ & 0.1 \\
5 & 10 & 10 & 0.1 & $4.004 \mathrm{e}-4$ & $1.602 \mathrm{e}-3$ & 0.218 & $5.84 \mathrm{e}-13$ & $5.84 \mathrm{e}-11$ & 0.01 \\
\hline
\end{tabular}

this work. The matrix porosity and permeability were set to 0.02 and $1 \times 10^{-18} \mathrm{~m}^{2}$ respectively, while the fracture porosity was assigned uniformly at an initial value of 0.5 . Table 2 indicated the predefined scenarios with various magnitudes of initial permeability anisotropy. The fracture aperture was kept identical for each element in the model, while the fracture spacing was varied for each axis.

\subsection{Thermal front propagation}

With the extraction of thermal energy from original host rock, there will be a temperature gradient profile in the spatial of reservoir, which is displayed as a response of the thermal front migration. We investigated the influence of anisotropic permeability $(100,10,1,0.1$, and 0.01$)$ on thermal front propagation from the injection well to the withdrawal well. The two bound anisotropy values $\eta$ are prescribed as top bound 100 and low bound 0.01 , obtained by varying the fracture spacing in the $x$ and $z$ directions respectively. The initial fracture aperture was $4.0 \times 10^{-4} \mathrm{~m}$.

Fig. 3 shows the migration of the thermal front within the reservoir as anisotropic permeability varied. When the anisotropic permeability was larger than 1 , the horizontal $x$ direction provided the dominating flow channel, higher flow velocity will facilitate heat depleting in horizontal $x$ direction. On the other hand, when anisotropic permeability was 0.1 and 0.01 , the conductive heat transfer was more active along the $z$-axis. The migration of the thermal front across the reservoir for anisotropic values of 100 and 10 (Figs. 3a and 3b) is faster, compared to the migration of the thermal front for anisotropic values of $1,0.1$ and 0.01 (Figs. 3c, 3d and 3e). The time of thermal breakthrough at production well for each case reflects the speed of advancement of the thermal front. The quick thermal front propagation for the anisotropic value of 100 resulted in an early thermal breakthrough (Fig. 3f) at the producer well at $5.0 \times 10^{6} \mathrm{~s}(2$ months $)$. Anisotropic permeability value of 1 , showed the slowest rate of thermal front propagation and the case with the most delayed thermal breakthrough at $3.5 \times 10^{7} \mathrm{~s}$ (1.1 years).

Fig. $3 \mathrm{c}$ with anisotropic permeability value of 1 , shows the lowest thermal front gradients, while Fig. 3e with anisotropic permeability value of 0.01 (Case 5) showed the steepest thermal front gradient. The high thermal gradient of case 5 represents a more desirable situation for an economically viable geothermal reservoir. This indicates the amount of heat transfer from the rock matrix to the fractures (Bagalkot and Kumar, 2015). High thermal gradient suggests high heat transfer from the rock matrix to the fractures, with a more depletion of thermal energy from surrounding host rocks. Conversely, a low thermal gradient across the reservoir means the reservoir temperature is cooling down simutanneously, indicating low heat transfer efficiency from the local rock matrix to the fractures. However, the thermal gradient in Fig. 3e reduced and resulted in a non-uniform sweep across the reservoir. This non-uniform sweep resulted in an early breakthrough of $10^{7} \mathrm{~s}$ (4 months).

The non-uniform thermal transfer in high permeable channel from injector to producer will inevitably generate to the localized cooling response. Fig. 4 shows the cooling regime comparison for each case scenario at a different simulation 


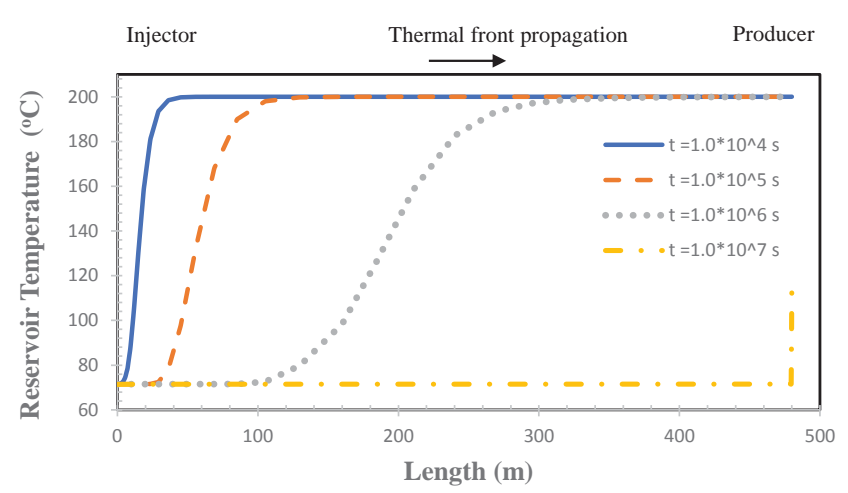

(a)

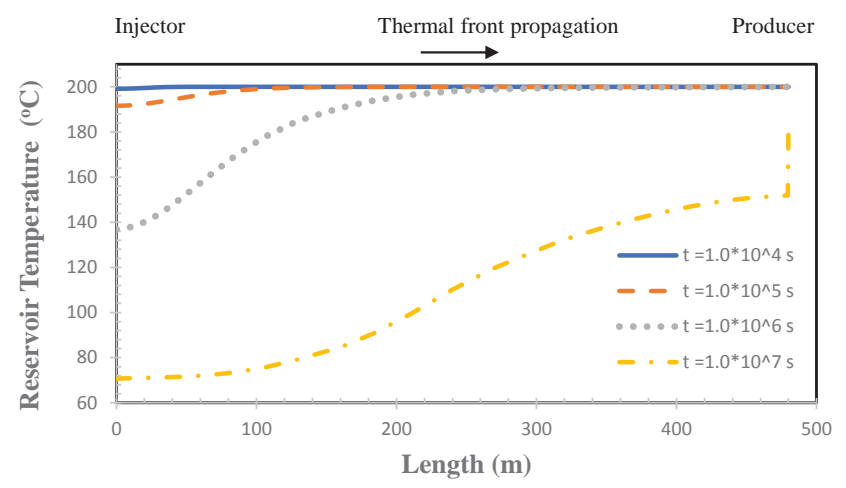

(c)

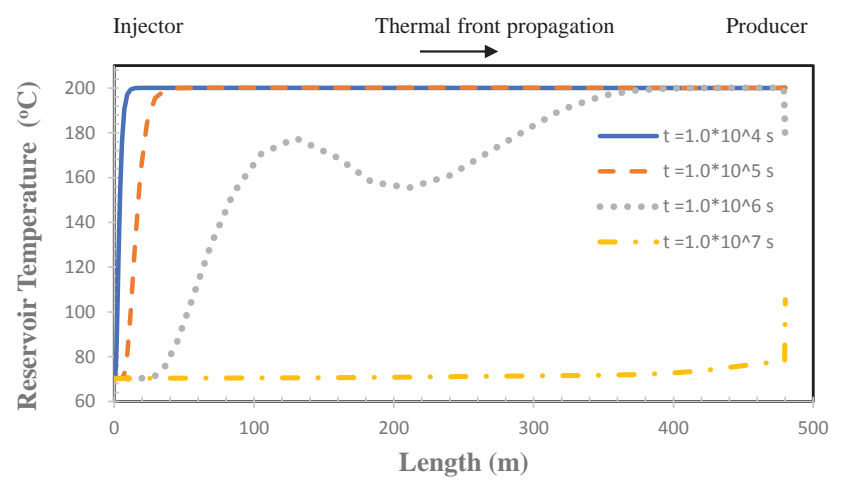

(e)

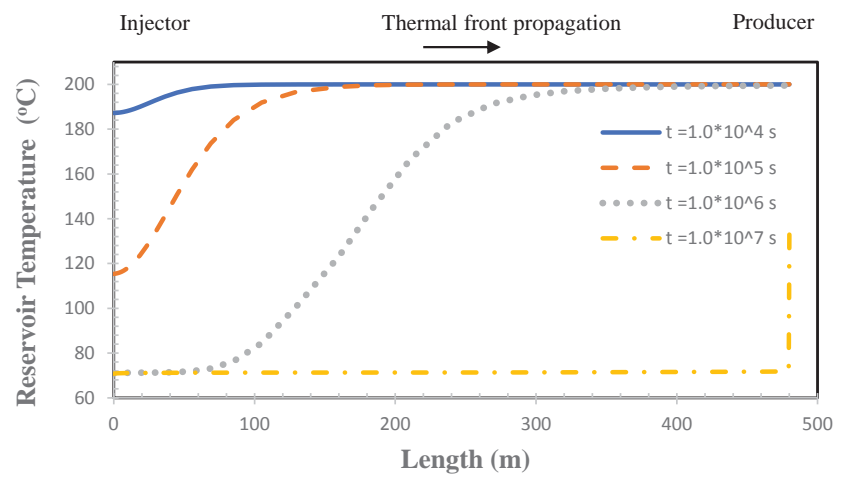

(b)

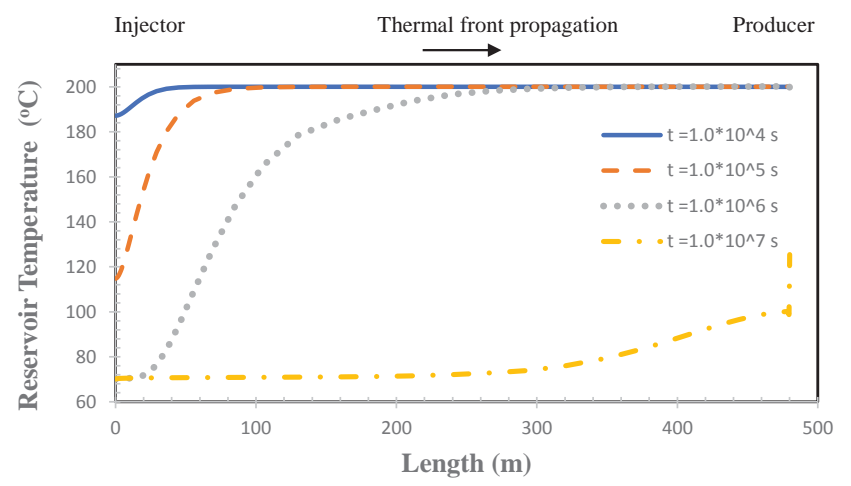

(d)

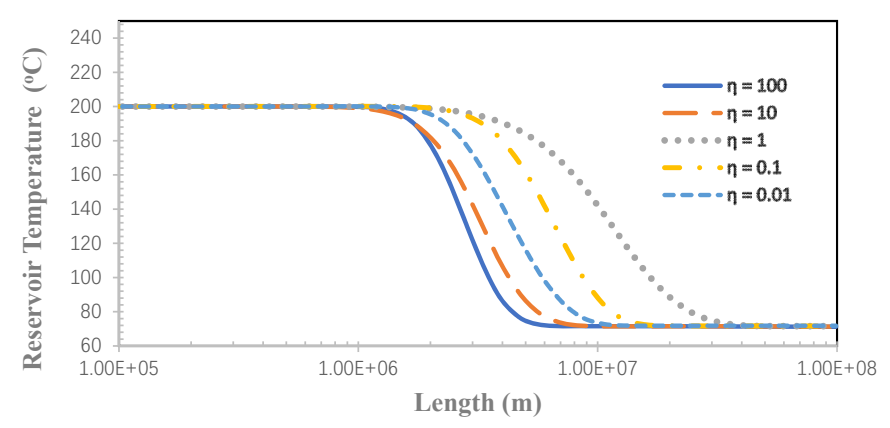

(f)

Fig. 3. Reservoir temperature evolution under (a) $\eta=100$, (b) $\eta=10$, (c) $\eta=1$, (d) $\eta=0.1$, and (e) $\eta=0.01$. (f) Transient advance of temperature within the reservoir at the withdrawal well.

timing. The contour of rock temperature with time elapsed demonstrated that the permeability anisotropy could affect the propagation direction of thermal front and also the area of thermal drawdown. At large values of $\eta$ (Figs. 4a and $4 \mathrm{~b}$ ), the cooling regimes were majorly migrating in the $\mathrm{x}$ direction due to the preferential fluid flow in the $\mathrm{x}$-direction. Anisotropic permeability value of 1 (Fig. 4c), generated a spherical cooling regime that expanded uniformly along the $x$ and $z$-axes. At lower values of $\eta$ (Figs. $4 \mathrm{~d}$ and $4 \mathrm{e}$ ), preferential fluid flow occurred in the $z$-direction and the cooling rock propagated in the $z$-direction and gradually migrated toward the $\mathrm{x}$-direction. This resulted in a wider sweep area, compared to larger anisotropic values. The wide sweep area of anisotropic values of 0.1 and 0.01 in the vertical directions indicates an improved comprehensive sweep efficiency, and the cooling area evolution suggests that scenarios with low anisotropic values could absorb more heat energy from the the reservoir than the scenarios with high permeability anisotropy values $(100,10$, and 1$)$, when the injected fluid is directly circulated from injection well to production well is normal to highest permeability direction.

\subsection{Anisotropic permeability evolution}

The resulted various modes of thermal front propagation within the reservoir could further alter the evolution of perme- 


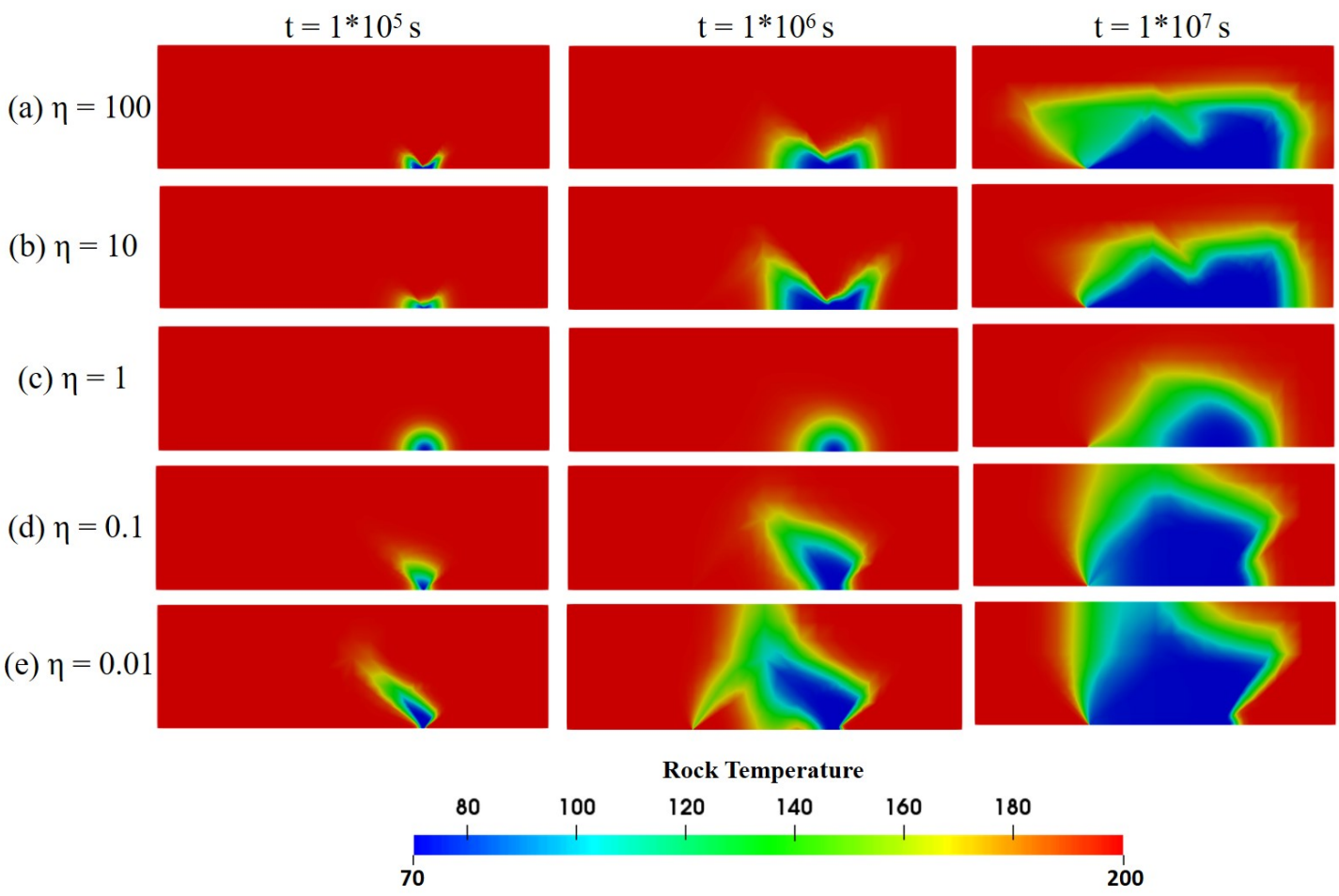

Fig. 4. Contour map of rock temperature distribution at $t=1.0 \times 10^{5} \mathrm{~s}, 1.0 \times 10^{6} \mathrm{~s}, 1.0 \times 10^{7} \mathrm{~s}$, respectively, for (a) $\eta=100$, (b) $\eta=10$, (c) $\eta=1$, (d) $\eta=0.1$, and (e) $\eta=0.01$.

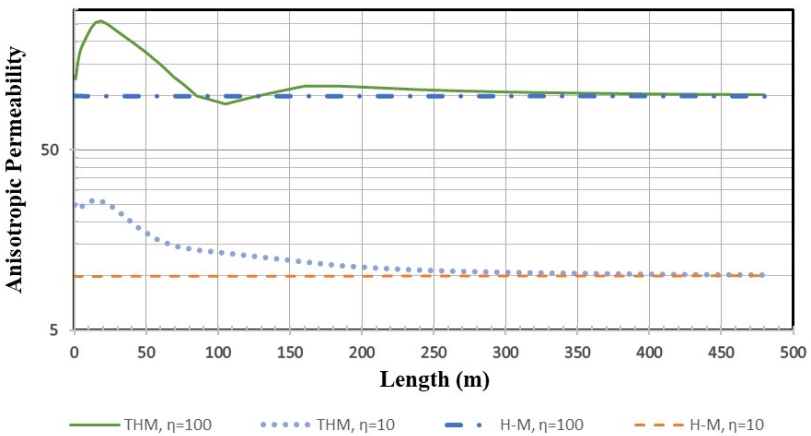

(a)

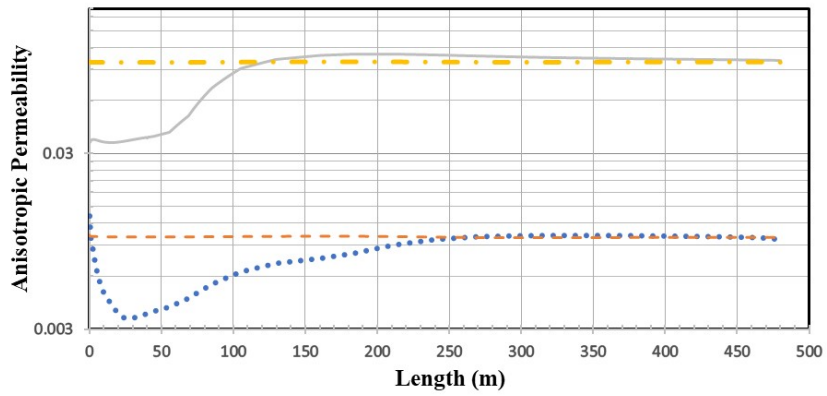

— THM, $n=0.1 \quad \cdots \cdots T$ THM, $n=0.01 \quad \cdots H-M, n=0.1 \quad \cdots--H-M, n=0.01$

(b)

Fig. 5. Anisotropic permeability evolution from injector to withdrawal well for (a) anisotropic values of 100 and 10 at $4.7 \times 10^{5} \mathrm{~s}$, and (b) anisotropic values of 0.1 and 0.01 at $4.7 \times 10^{5} \mathrm{~s}$.

ability anisotropy from the injector to withdrawal well. Fig. 5 confirms that the influence of hydraulic and mechanical forces on changing fracture permeability evolution is minimal, compared to the impact of thermal forces. The thermal force influenced the anisotropic permeability significantly based on the fully coupled thermal-hydraulic-mechanical components. The anisotropic permeability values with initial 100 and 10 increased by a factor of 2.5 , while there is a very marginal increase for anisotropic permeability value of 1 . In the scenarios with small permeability anisotropy magnitude, the permeability anisotropy ratio decreased by a factor of 0.35 , indicated the reduction of permeability in the horizontal $x$ direction. This normalised ratio of the peak anisotropic permeability over the initial anisotropic permeability illustrates that the induced thermal stress from cooling rocks can substantially change the permeability of particular locality in the reservoir, where is subjected to the highest influence of thermal drawdown and gradient.

The increased anisotropic permeability at $\eta=100$ and $\eta$ $=10$ indicated the preferential fluid flow occurred in the $\mathrm{x}$ direction, as permeability in the $\mathrm{x}$-direction is predominant. The compressive normal stress acting on the x-axis $\sigma_{x x}$ (Fig. 6a) was reduced, whereas the compressive stress is increased along the $z$-axis (Fig. 6b). This suggests that the cooling rocks imposed an unloading effect on the injection adjacent areas in the $x$ direction, while the surrounding hot reservoir rocks expand along the $z$-axis (Sandwell, 1986). The reduction in compressive stress allowed for an increase in fracture aperture 


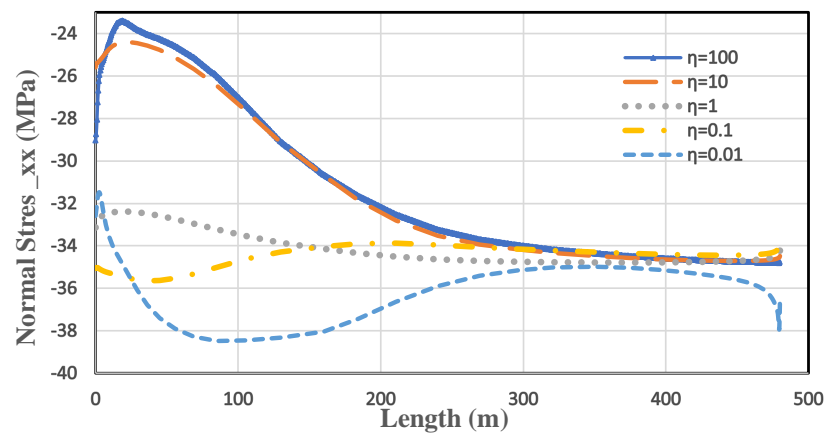

(a)

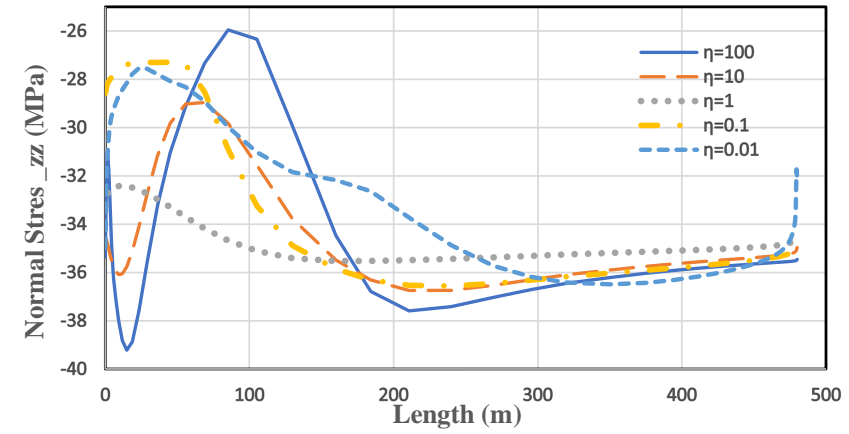

(b)

Fig. 6. (a) evolution of normal stress $\sigma_{x x}$ from injector to withdrawal well at $4.7 \times 10^{5} \mathrm{~s}$, and (b) evolution of normal stress $\sigma_{x x}$ from injector to withdrawal well at $4.7 \times 10^{5} \mathrm{~s}$.

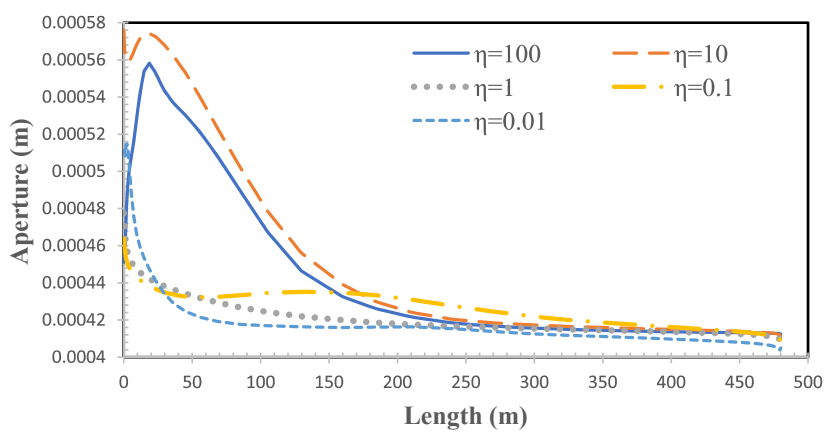

(a)

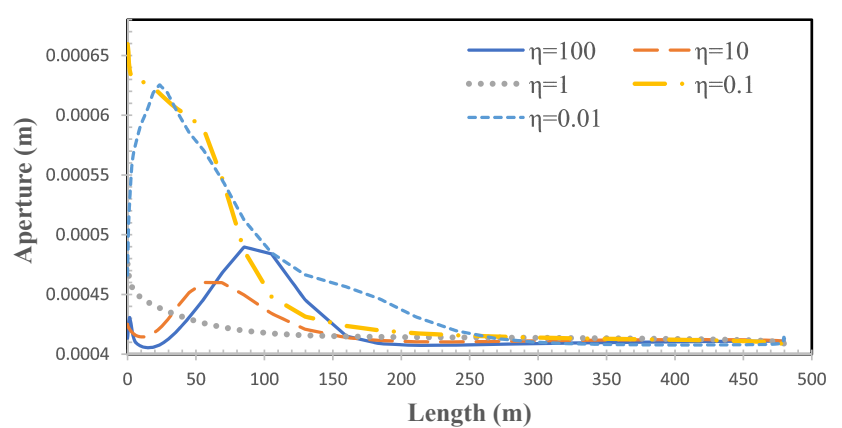

(b)

Fig. 7. (a) evolution of fracture aperture along the $\mathrm{x}$-axis from injector to withdrawal well at $4.7 \times 10^{5} \mathrm{~s}$, and (b) evolution of fracture aperture along the $z$-axis from injector to withdrawal well at $4.7 \times 10^{5} \mathrm{~s}$.

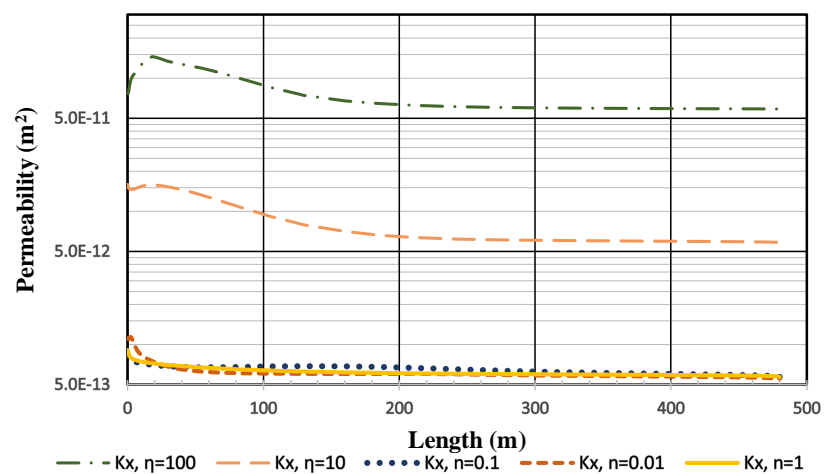

(a)

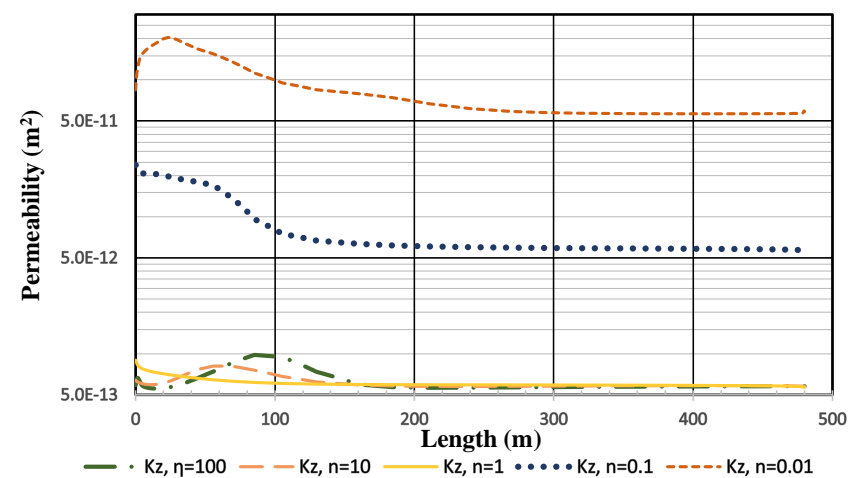

(b)

Fig. 8. (a) evolution of permeability $K_{x}$ from injector to withdrawal well at $4.7 \times 10^{5} \mathrm{~s}$ along the $\mathrm{x}$-axis, and (b) evolution of permeability $K_{z}$ from injector to withdrawal well at $4.7 \times 10^{5} \mathrm{~s}$ along the $z$-axis. 


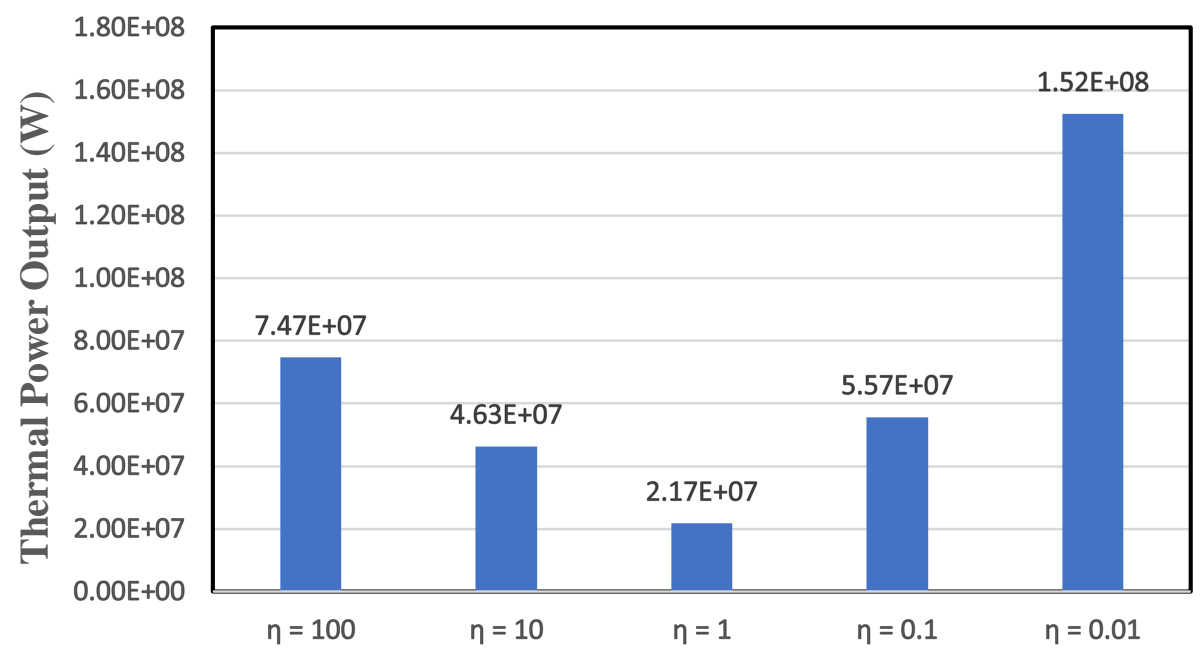

Fig. 9. Thermal power generation for different anisotropic permeabilities at time $1.09 \times 10^{8} \mathrm{~s}$.

(Fig. 7a) and invariably an increase in fracture permeability $k_{x}$ (Fig. 8a) in the x-direction. Similarly, the increase in compressive stress in the $z$-axis resulted in a decrease in fracture aperture (Fig. 7b) and permeability reduction (Fig. $8 b)$.

However, the compressive stress acting on the $z$-axis direction is reduced away from the injector well $(>20$ $\mathrm{m})$ with anisotropy values of 100 and 10 . This observation could be illustrated by the Figs. $6 \mathrm{a}$ and $6 \mathrm{~b}$, indicating the start of simultaneous propagation of cold rock and thermal unloading in the $z$-axis reducing the normal stress. The reduced compressive stress in both $x$ and $z$-axes prompted an early thermal breakthrough for scenarios with anisotropic values of 100 and 10, due to the high permeability in $x$ direction. The degree of unloading in the $z$-axis enhanced the velocity of thermal front propagation, as the fact of thermal unloading in the x-direction for both cases is approximately equal (Fig. 6a). For $\eta=100$ and $\eta=10$, the compressive stress is reduced to $26 \mathrm{MPa}$ and $29 \mathrm{MPa}$ respectively, and early thermal breakthrough corresponds to the anisotropic value of 100 . The subsequent decrease in the compressive stress along the $z$ axis was accompanied by an increase in fracture aperture and permeability along the $z$-axis. Decrease and increase in fracture permeability along the $z$-axis and $\mathrm{x}$-axis respectively is also indicative of rock expansion along the $z$-axis and rock contraction along the $\mathrm{x}$-axis.

Inversely, the decrease in anisotropic permeability at $\eta$ $=0.1$ and $\eta=0.01$ resulted from an increase in the $z$ permeability and a decrease in the x-permeability. Fluid flow occurred mainly in the $z$-direction and resulted in the propagation of cold rocks along the $z$-axis. Rock contraction along the $z$-axis reduced the compressive stress acting on the $z$-axis (Fig. 7b). Meanwhile, the compressive stress acting on the $\mathrm{x}$-axis is increased due to rock expansion along the $\mathrm{x}$-axis (Fig. 7a). This suggests that the degree of loading in the $x$ axis determines the velocity of thermal front propagation for anisotropic values of $\eta=0.1$ and $\eta=0.01$. For $\eta=0.1$ and $\eta$ $=0.01$, the compressive stress is increased to $35 \mathrm{MPa}$ and 38
MPa respectively, and early thermal breakthrough corresponds to the anisotropic value of 0.01 . The decreased compressive stress along the $z$-axis resulted in an enhancement of fracture aperture in the $z$-axis (Fig. 7b). Conversely, the increased compressional stress along the $\mathrm{x}$-axis produced a decrease in fracture aperture (Fig. 7a) and fracture permeability $k_{x}$ (Fig. $8 a)$ in the $x$-axis.

\subsection{Thermal power output}

The rate of thermal energy production $W_{e}$ is defined as (Gan and Elsworth, 2014b):

$$
W_{e}=Q \Delta H
$$

where $Q$ is the mass flow rate $(\mathrm{kg} / \mathrm{s})$, and $\Delta H$ is the enthalpy difference between the injected water and produced water. Fig. 9 shows the thermal power output for each anisotropic permeability scenario by the time $1.09 \times 10^{8} \mathrm{~s}$ (3.46 years). Anisotropic value of 0.01 (Case 5) generated the highest power output with a value of $152 \mathrm{MW}$, and anisotropic value of 100 produced an output of $74.7 \mathrm{MW}$. On the other hand, the anisotropic value of 1 generated the least power output (21.7 MW), with a percentage difference of $85.72 \%$ from output generated from Case 5.

The generated thermal power output for each case corresponds to their sweep area (Fig. 5). Wider sweep area $(\eta=$ $0.01)$ results in an improved sweep efficiency and high energy generation while smaller sweep area $(\eta=1)$ results in reduced sweep efficiency and low energy generation. However, this is not always true. Despite the fact the anisotropic value of 0.1 has a broader sweep area than the anisotropic value of 100 , the thermal output for $\eta=100$ (74.7 MW) is greater than that of $\eta=0.1(55.7 \mathrm{MW})$. This suggests that permeability anisotropy influences the ultimate amount of power generation.

\section{Conclusion}

In this work, the influence of anisotropic permeability was 
explored by examining the thermal front propagation velocity, evolution of permeability, and resulted heat sweep efficiency. Low anisotropic permeability returns an uniform thermal front propogation across reservoir, and a more comprehensive sweep efficiency, compared to high anisotropic permeability scenarios. Hence, creating the flow circulation direction from the injector to withdrawal well normal to the high permeability channel is beneficial in geothermal engineering designs, in order to achieve higher sweep efficiency. In addition, the comparison of fully THM (Thermal-Hydraulic-Mechanical) coupling simulations against the thermal decoupling scenarios revealed that the induced thermal stress significantly altered the stress state of fractures, and thereby affect the heat transfer efficiency in return. Depending on the direction of cold fluid circulation due to the permeability anisotropy, the resulted induce thermal stress will reduced the normal stress state in different direction, as the injection of cold fluid cools the rock matrix and induce thermal shrinkage (Sandwell, 1986). In this work, the thermal unloading response for the low anisotropy scenarios decreased the extent of compaction on fracture plane in the sub-horizontal direction ( $z$ direction), thereby increase fracture aperture and permeability, while the thermal stress shrank the fracture laid align in the horizontal $x$ direction by increasing the normal stress. Consequently, the response of thermal unloading will also exaggerate the degree of anisotropy, by prompting the permeability of fracture at the direction of heat exchange.

\section{Acknowledgments}

The author would like to thank Dr. Quan Gan's supervision, family's support, and Prof. Cai's valuable suggestions in completing this work, as part of the MSc degree requirements in Reservoir Engineering programme in the University of Aberdeen.

Open Access This article is distributed under the terms and conditions of the Creative Commons Attribution (CC BY-NC-ND) license, which permits unrestricted use, distribution, and reproduction in any medium, provided the original work is properly cited.

\section{References}

Bagalkot, N., Kumar, G.S. Thermal front propagation in variable aperture fracture-matrix system: A numerical study. Sadhana 2015, 40(2): 605-622.

Bodvarsson, G. On the temperature of water flowing through fractures. J. Geophys. Res. 1969, 74(8): 1987-1992.

Bödvarsson, G.S., Tsang, C.F. Injection and thermal breakthrough in fractured geothermal reservoirs. J. Geophys. Res. Sol. Ea. 1982, 87(B2): 1031-1048.

Elsworth, D. Theory of thermal recovery from a spherically stimulated hot dry rock reservoir. J. Geophys. Res. Sol. Ea. 1989, 94(B2): 1927-1934.

Gan, Q., Elsworth, D. Analysis of fluid injection-induced fault reactivation and seismic slip in geothermal reservoirs: Seismic slip induced by thermal stress. J. Geophys. Res.Sol. Ea. 2014a, 119(4): 3340-3353.

Gan, Q., Elsworth, D. Thermal drawdown and late-stage seismic-slip fault reactivation in enhanced geothermal reservoirs: Thermal front propagation and fault slip. J.
Geophys. Res. Sol. Ea. 2014b, 119(12): 8936-8949.

Gan, Q., Elsworth, D. A continuum model for coupled stress and fluid flow in discrete fracture networks. Geomech. Geophys. Geo-Energy Geo-Resour. 2016a, 2(1): 43-61.

Gan, Q., Elsworth, D. Production optimization in fractured geothermal reservoirs by coupled discrete fracture network modeling. Geothermics 2016b, 62: 131-142.

Gringarten, A.C., Witherspoon, P.A., Ohnishi, Y. Theory of heat extraction from fractured hot dry rock. J. Geophys. Res. 1975, 80(8): 1120-1124.

Itasca. FLAC3D: Fast lagrangian analysis of continua in 3 dimensions-version 4.0. Minneapolis, Minnesota: Itasca Consulting Group, 2009.

Jahediesfanjani, H., Civan, F. Improving performance of the naturally fractured carbonate reservoirs by means of various stimulation and completion techniques. Paper SPE 103986 Presented at the International Oil Conference and Exhibition, Cancun, Mexico, 31 August-2 September, 2006.

Kolditz, O., Clauser, C. Numerical simulation of flow and heat transfer in fractured crystalline rocks: Application to the Hot Dry Rock site in Rosemanowes (UK). Geothermics 1998, 27(1): 1-23.

Min, K.B., Rutqvist, J., Elsworth, D. Chemically and mechanically mediated influences on the transport and mechanical characteristics of rock fractures. Int. J. Rock Mech. Min. 2008, 46(1): 80-89.

Pruess, K. Heat transfer in fractured geothermal reservoirs with boiling. Water Resour. Res. 1983, 19(1): 201-208.

Pruess, K. The TOUGH codes-A family of simulation tools for multiphase flow and transport processes in permeable media. Vadose Zone J. 2004, 3(3): 738-746.

Rutqvist, J., Stephansson, O. The role of hydromechanical coupling in fractured rock engineering. Hydrogeol. J. 2003, 11(1): 7-40.

Sandwell, D.T. Thermal stress and the spacings of transform faults. J. Geophys. Res. 1986, 91(B6): 6405.

Snow, D.T. Anisotropie permeability of fractured media. Water Resour. Res. 1969, 5(6): 1273-1289.

Taron, J., Elsworth, D. Thermal-hydrologic-mechanicalchemical processes in the evolution of engineered geothermal reservoirs. Int. J. Rock Mech. Min. 2009, 46(5): 855-864.

Taron, J., Elsworth, D. Coupled mechanical and chemical processes in engineered geothermal reservoirs with dynamic permeability. Int. J. Rock Mech. Min. 2010, 47(8): 1339-1348.

Taron, J., Elsworth, D., Min, K.B. Numerical simulation of thermal-hydrologic-mechanical-chemical processes in deformable, fractured porous media. Int. J. Rock Mech. Min. 2009, 46(5): 842-854.

Watanabe, K., Takahashi, H. Fractal geometry characterization of geothermal reservoir fracture networks. J. Geophys. Res. Sol. Ea. 1995, 100(B1): 521-528.

Witherspoon, P.A., Wang, J.S.Y., Iwai, K., et al. Validity of Cubic Law for fluid flow in a deformable rock fracture. Water Resour. Res. 1980, 16(6): 1016-1024. 